\title{
Choline Inhibits Ischemia-Reperfusion- Induced Cardiomyocyte Autophagy in Rat Myocardium by Activating Akt/mTOR Signaling
}

\author{
Pengzhou Hang a, b Jing Zhao ${ }^{c}$ Zhenli Su ${ }^{a}$ Hanqi Sun ${ }^{a} \quad$ Tingting Chen ${ }^{a}$ \\ Lihui Zhao ${ }^{a}$ Zhimin Dua, \\ aDepartment of Pharmacy, the Second Affiliated Hospital of Harbin Medical University (Institute of \\ Clinical Pharmacy, the Heilongjiang Key Laboratory of Drug Research, Harbin Medical University), \\ Harbin, bDepartment of Clinical Pharmacology, College of Pharmacy, Harbin Medical University, Harbin, \\ 'Department of Cardiology, the First Affiliated Hospital of Harbin Medical University (Key Laboratory of \\ Cardiac Diseases and Heart Failure, Harbin Medical University), Harbin, P. R. China
}

\section{Key Words}

Ischemia-reperfusion injury • Autophagy $\bullet$ Choline $•$ Akt $•$ mTOR

\begin{abstract}
Backgroud/Aims: Growing evidence suggests that both cardiomyocyte apoptosis and excessive autophagy exacerbates cardiac dysfunction during myocardial ischemia-reperfusion (IR). As a precursor of acetylcholine, choline has been found to protect the heart by repressing ischemic cardiomyocyte apoptosis. However, the relationship between choline and cardiomyocyte autophagy is unclear. The present study aimed to investigate whether autophagy was involved in the cardioprotection of choline during IR. Methods: Rats were subjected to $30 \mathrm{~min}$ reversible ischemia by ligation of left anterior descending coronary artery followed by reperfusion for $2 \mathrm{~h}$. Choline $(5 \mathrm{mg} / \mathrm{kg}$, i.v.) alone or along with rapamycin $(5 \mathrm{mg} /$ $\mathrm{kg}$, i.p.) were injected $30 \mathrm{~min}$ before ischemia. Transmission electron microscopy, hematoxylin and eosin (HE) and TUNEL staining were conducted to evaluate the effect of choline on cardiac apoptosis and autophagy. Protein levels of autophagic markers including LC3, beclin-1 and p62 as well as Akt and mammalian target of rapamycin (mTOR) were examined by Western blotting. Results: Myocardial IR-induced cardiac apoptosis and accumulation of autophagosomes was attenuated by choline. Choline treatment significantly ameliorated myocardial IR-induced autophagic activity characterized by repression of beclin-1 over-activation, the reduction of autophagosomes, the LC3-II/LC3-I ratio, and p62 protein abundance. In addition, IR-induced downregulation of $\mathrm{p}-\mathrm{Akt} / \mathrm{mTOR}$ cascade was increased by choline. However, the above functions of choline were abolished by rapamycin. Conclusion: These findings suggest that
\end{abstract}

P. Hang and J. Zhao contributed equally to this paper.

Zhimin $\mathrm{Du}, \mathrm{PhD}$

and Pengzhou Hang, PhD

KARGER
Department of Pharmacy, the Second Affiliated Hospital of Harbin Medical University Xuefu Road 246\#, Nangang District, Harbin 150086, (P. R. China);

Tel.+8645186605353. E-Mail dzm1956@126.com, hangpengzhou@163.com 


\section{Cellular Physiology Cell Physiol Biochem 2018;45:2136-2144

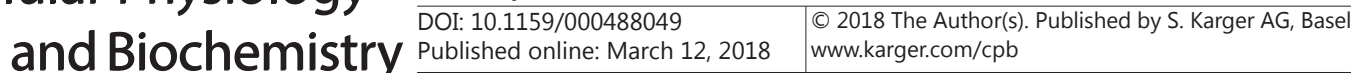 \\ Hang et al.: Choline Mitigates IR-Induced Autophagy}

choline plays a protective role against myocardial IR injury by inhibiting excessive autophagy, which might be associated with the activation of Akt/mTOR pathway. This study provides new mechanistic understanding of cardioprotective effect of choline and suggests novel potential therapeutic targets for cardiac IR injury.

(C) 2018 The Author(s)

Published by S. Karger AG, Basel

\section{Introduction}

Myocardial ischemia-reperfusion (IR) injury occurs frequently in percutaneous coronary intervention and several cardiac surgeries in clinical practice, which is the primary contributor to the morbidity and mortality of coronary artery diseases [1,2]. Previous studies documented that cardiomyocyte apoptosis and necrosis play dominant roles in IR injury [3]. Recently, accumulating evidence suggests that autophagy, also called type II programmed cell death, plays an important role in IR injury $[4,5]$. Autophagy is well known as an intracellular lysosomal self-degrading process to remove and recycle long-lived proteins and damaged organelles. In detail, autophagy occurs at basal level is used for degradation of damaged or unnecessary proteins to maintain cellular homeostasis. In contrast, excessive autophagy is responsible for self-destruction and ultimately causes autophagic cell death, accompanied by the upregulation of microtubule-associated protein 1 light chain 3 (LC3) and beclin-1 [6]. It has been revealed that dysregulated autophagy is closely related to various cardiac diseases such as myocardial infarction [7], myocardial IR injury [8], and cardiac hypertrophy [9]. A variety of studies have suggested that myocardial ischemia induces autophagy through an AMP-induced protein kinase (AMPK)-dependent mechanism. In contrast, myocardial IR activates autophagy mainly by a beclin-1-dependent mechanism. It has also been reported that AMPK-induced autophagy in myocardial ischemia process is protective, whereas beclin1-mediated autophagy in reperfusion period is detrimental [10,11]. Thus, inhibition of beclin- 1 and repression of excessive cardiomyocyte autophagy is considered to be positive in prevention of IR injury [12]. Accordingly, appropriate interventions for regulating autophagy are anticipated to limit the IR injury and improve cardiac function.

$\mathrm{PI} 3 \mathrm{~K} / \mathrm{Akt} / \mathrm{mammalian}$ target of rapamycin (mTOR) signaling pathway plays a central and extensive role in the biological activity of cells especially cross-talk between apoptosis and autophagy [13]. Evidence also indicates that activation of PI3K/Akt/mTOR pathway negatively regulated autophagy characterized by downregulation of autophagic marker LC3 $[14,15]$. For example, nerve growth factor protected ischemic heart by inhibition of autophagy related proteins via activating PI3K/Akt/mTOR pathway [16]. Conversely, inhibition of mTOR signaling with rapamycin was found to induce autophagy. For instance, Wang et al. reported that rapamycin partially abolished the salutary effect of basic fibroblast growth factor in myocardial I/R by inducing autophagy [17].

As a precursor molecule of neurotransmitter acetylcholine, studies have clarified that choline exhibited beneficial effects on several cardiac diseases including myocardial infarction [18], ischemic arrhythmias [19], IR injury [20] and cardiac hypertrophy [21]. Meanwhile, previous studies have revealed that choline reduced infarct size and prevented ischemic arrhythmias by inhibiting cardiomyocyte apoptosis as well as preserving phosphorylated connexin43 and regulating ion channels including L-type $\mathrm{Ca}^{2+}$ channel and $\mathrm{Na}^{+} / \mathrm{Ca}^{2+}$ exchanger [18-20]. Even though, the mechanisms for the function of choline are not fully understood. The direct relationship between choline and cardiomyocyte autophagy is poorly understood. Based on these findings, the present study established rat myocardial IR model to explore the role and mechanism of choline-mediated autophagy in response to myocardial IR injury. 


\section{Cellular Physiology Cell Physiol Biochem 2018;45:2136-2144 \begin{tabular}{ll|l} 
and Biochemistry Publisned onIIne: IVIarch 12, 2018 & $\begin{array}{l}\text { (c) } 2018 \text { The Author(s). Published by S. Karger AG, Basel } \\
\text { www.karger.com/cpb }\end{array}$
\end{tabular}}

\section{Materials and Methods}

\section{Cardiac IR modeling}

Healthy adult Wistar rats (male, 180-220 g, provided by Animal Center of the Second Affiliated Hospital of Harbin Medical University) were used. All experiments were approved by Ethical Committee of Harbin Medical University and in accordance with the Guideline for the Care and Use of Laboratory Animals published by the US National Institutes of Health (8th edition, 2011). Experimental protocol was shown in Fig. 1. Totally, twenty rats were randomly divided into the following four groups: (1) sham-operated group (Sham, $n=5) ;(2)$ IR group ( $n=5) ;(3)$ IR with choline treatment (Cho, $n=5$ ); (4) IR with choline and rapamycin treatment (Cho+Rapa, n=5). Rats were anesthetized with pentobarbital sodium $(40 \mathrm{mg} / \mathrm{kg})$ and subjected to reversible left anterior descending coronary artery for $30 \mathrm{~min}$ and then reperfusion for $2 \mathrm{~h}$ to establish IR model in group (2). Rats in group (1) were not occluded. In groups (3), the rats were injected with choline chloride (5 mg/ $\mathrm{kg}$ ) via tail vein $30 \mathrm{~min}$ before ischemia. In groups (4), the rats were injected with choline chloride $(5 \mathrm{mg} / \mathrm{kg}$ ) via tail vein and rapamycin (5 mg/ $\mathrm{kg}$ ) intraperitoneally $30 \mathrm{~min}$ before ischemia. Then, rats were sacrificed and ischemic border zones were collected for subsequent experiments.

\section{Hematoxylin-eosin (HE) staining and transmission electron microscopy}

To examine the morphological changes, ischemic border zones of rat left ventricles were quickly dissected and immersed in 10\% neutral buffered formalin for $24 \mathrm{~h}$ and stained with HE. In addition, to detect the quantity of autophagosome, transmission electron microscopy was performed as previously described [22]. Ischemic border zones of rat left ventricles were cut into ultra-thin sections. Then, these sections were fixed in $2.5 \%$ glutaraldehyde in $0.1 \mathrm{~mol} / \mathrm{L} \mathrm{PBS} \mathrm{(pH} \mathrm{7.4),} \mathrm{stained} \mathrm{in} \mathrm{uranyl} \mathrm{acetate} \mathrm{and} \mathrm{dehydrated} \mathrm{in}$ ethanol. Following this, they were embedded in epoxy resin by standard operations and observed with a transmission electron microscope (JEM-1220, JEOL Ltd., Tokyo, Japan).

\section{TdT-mediated dUTP nick end labeling (TUNEL) staining}

TUNEL staining was used to detect the cardiomyocyte apoptosis in ischemic border zones of left ventricles as mentioned in our previous work [22, 23] using a TUNEL fluorescence FITC kit (Roche, Indianapolis, USA) according to the manufacturer's manual. After staining, the slices were immerged into DAPI (1:30, Beyotime Biotechnology, Haimen, China) solution to stain nuclei for $5 \mathrm{~min}$. Fluorescence staining was viewed by microscope (Olympus, BX-60, Tokyo, Japan). The apoptotic rate was calculated as percentage of TUNEL-positive cells per field.

\section{Western blotting}

Total protein was extracted from ischemic border zone of rat left ventricles for western blotting analysis as described previously [24]. SDS-PAGE was conducted to separate protein and further detect corresponding protein expression. Membranes were incubated with primary antibodies against p-Akt (Ser473, 1:1000), pan-Akt (1:1000), p-mTOR (Ser2448, 1:1000), mTOR (1:1000), LC3 (1:1000), beclin-1 


\section{Cellular Physiology Cell Physiol Biochem 2018;45:2136-2144 \begin{tabular}{l|l} 
and Biochemistry Published online: March 12, 2018 & $\begin{array}{l}\text { (c) } 2018 \text { The Author(s). Published by S. Karger AG, Basel } \\
\text { www.karger.com/cpb }\end{array}$
\end{tabular} \\ Hang et al.: Choline Mitigates IR-Induced Autophagy}

(1:500), p62 (1:1000), GAPDH (1:1000) or $\beta$-tubulin $(1: 1000)$ overnight at $4{ }^{\circ} \mathrm{C}$, followed by incubation with fluorescence-labeled secondary goat-anti-rabbit or goat-anti-mouse antibody (IRDye 800CW, LI-COR Biosciences, Lincoln, NE, USA). The images were scanned by Odyssey CLx Infrared Imaging System (LI-COR Biosciences, Lincoln, NE, USA). Band densities were calculated by Odyssey CLx v2.1 software and normalized to GAPDH or $\beta$-tubulin as a loading control.

\section{Reagents}

Choline chloride was purchased from Sigma (St. Louis, MO, USA, purity $\geq 98 \%$ ). Rapamycin was obtained from Solarbio Science \& Technology Co. Ltd (Beijing, China, purity 98\%). Anti-LC3 antibody was provided by Sigma (St. Louis, MO, USA). Anti-p-Akt (Ser473), anti-pan-Akt, anti-p-mTOR and anti-mTOR antibodies were provided by Cell Signaling Technology (Danvers, MA, USA). Anti-beclin-1 antibody was purchased from Abclonal (Wuhan, China). Anti-p62/SQTM1 antibody was purchased from Abcam (Dallas, USA). Anti-GAPDH antibody was purchased from ZSGB-BIO (Beijing, China). Anti- $\beta$-tubulin antibody was provided by Absin Bioscience Inc. (Shanghai, China).

\section{Statistical analysis}

All values were presented as Mean \pm SEM. Statistical differences were assessed with one-way ANOVA for multiple-groups with Graphpad 5.0 software (San Diego, CA, USA). Bonferroni post-hoc test was performed after ANOVA. $\mathrm{P}<0.05$ was considered to be statistically significant.

\section{Results}

Choline protects against cardiac IR injury

Previous studies by our laboratory and others have reported the protective role of choline against both ischemia insult and myocardial IR-induced arrhythmias [1820]. First of all, HE staining experiment was conducted to validate the protective effect of choline on cardiac IR injury. As displayed in Fig. 2A, cardiomyocytes fibers of myocardial IR rat ventricles were markedly damaged compared to sham rats, which was alleviated by choline. However, the beneficial effect of choline was reversed by rapamycin. Besides, cardiomyocyte apoptosis was also examined. As shown in Fig. 2B, apoptotic cells in myocardial IR rats were significantly higher than sham rats. Choline treatment markedly inhibited cardiomyocyte apoptosis, which was restored by rapamycin (Fig. 2D). These findings indicated that protective effect of choline could be reversed by rapamycin in myocardial IR rats.

\section{Choline inhibits cardiac IR-induced autophagy}

The above findings found that rapamycin could reverse the protective effect of choline, which suggested that autophagy is probably involved in the function of choline. So, we further investigated the changes of autophagic activity. Firstly, transmission

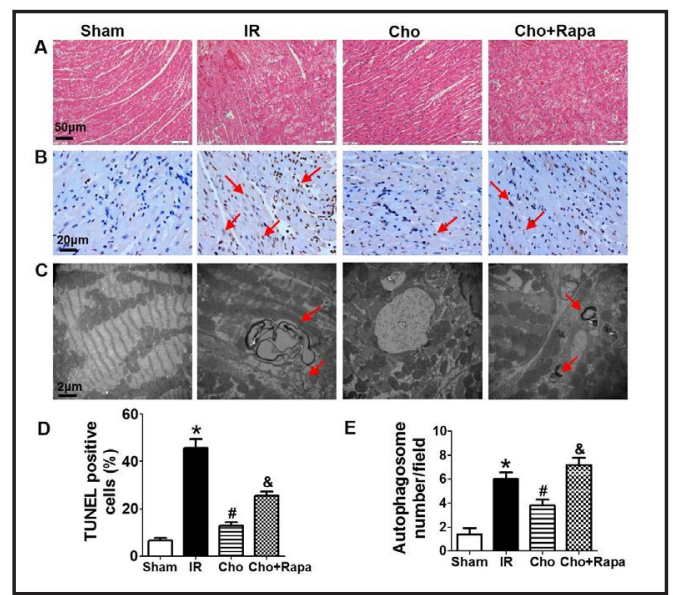

Fig. 2. Effect of choline and rapamycin on morphological changes, apoptosis and autophagy of rat left ventricular myocytes in response to IR. It was found that choline significantly inhibited cardiomyocyte apoptosis and autophagy in IR, and was blocked by rapamycin. (A) Representative images of haematoxylin and eosin (HE) staining from rat left ventricles, scale bar: $50 \mu \mathrm{m}$. (B) TUNEL staining pictures, scale bar: $20 \mu \mathrm{m}$. (C) Transmission electron microscope photographs showed the ultrastructure changes and autophagosome of rat left ventricular myocytes, the magnification is $12,000 \times$, scale bar: $2 \mu \mathrm{m}$. (D) Ratio of TUNEL-positive cells (stained in brown) per field. (E) Quantitative analysis of autophagosomes per field. ${ }^{*} \mathrm{P}<0.05$ vs. sham, $\# \mathrm{P}<0.05$ vs. $\mathrm{IR}, \& \mathrm{P}<0.05$ vs. Cho, $\mathrm{n}=5$ each group. 
Fig. 3. Protein expression of classical autophagic markers including LC3, beclin-1 and p62. It was found that protein levels of LC3 II , beclin-1 and p62 were increased in response to IR. Choline markedly repressed the expression of these proteins, which was reversed by rapamycin. (A) Representative western blot bands of LC3. LC3 bands comprise LC3-I at $18 \mathrm{kDa}$ and LC3-II at 16 kDa. (B) Statistical results of LC3-II/LC-3-I. (C) Representative western blot bands of beclin-1. (D) Statistical results of beclin-1 with GAPDH as internal control. (E) Representative western blot bands of p62. (F) Statistical results of p62 with GAPDH as internal control. ${ }^{*} \mathrm{P}<0.05$ vs. sham, $\# \mathrm{P}<0.05$ vs. IR, $\& \mathrm{P}<0.05$ vs. Cho, $n=5$ each group.

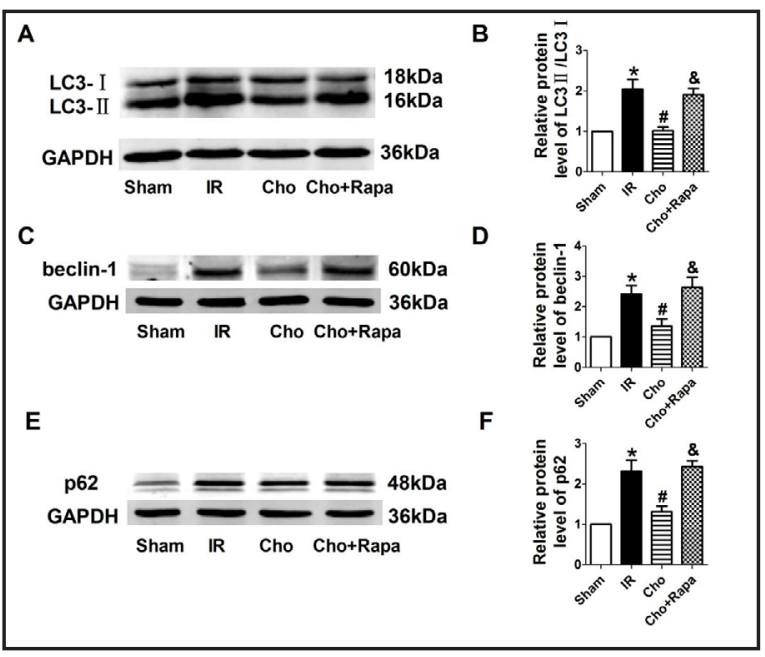

electron microscopy was used to detect the autophagosome in cardiomyocytes. As displayed in Fig. 2C, autophagosome was accumulated in IR rat ventricular myocytes. In contrast, the number of autophagosome was significantly reduced in choline group, which was abolished by rapamycin (Fig. 2E). We further validated protein expression of autophagic markers including LC3, beclin-1, and p62. Here we found that both LC3-II and beclin-1 expression was significantly increased in myocardial IR rats compared with sham rats, which was depressed by choline. However, the effects of choline were restored by rapamycin (Fig. 3A-D). Furthermore, myocardial IR-induced p62 upregulation was reduced in choline rats, which was reversed by rapamycin (Fig. 3E, F). Collectively, these data suggested that autophagosomes were accumulated and their degradation was impaired in myocardial IR, which was restored by choline.

\section{Choline activates Akt/mTOR pathway}

As rapamycin is a specific inhibitor of mTOR, protein expression of p-mTOR and mTOR was examined. As shown in Fig. 4C, protein level of p-mTOR was lower in myocardial IR rats than sham rats, and was significantly increased by choline. Rapamycin abolished the effect of choline on p-mTOR. No significant difference of

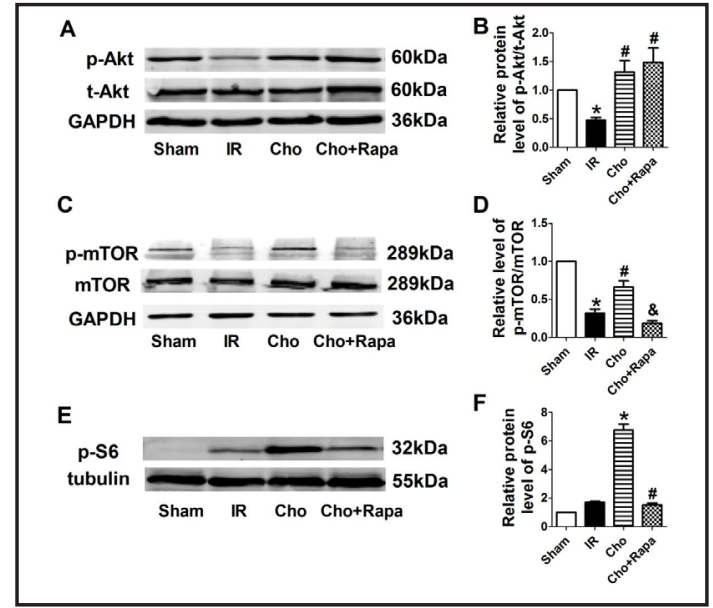

Fig. 4. Protein levels of Akt, mammalian target of rapamycin (mTOR) and phospho-S6 (p-S6). It was shown that protein expression of $\mathrm{p}$-Akt and $\mathrm{p}-\mathrm{mTOR}$ were downregulated in IR rats and upregulated by choline. Protein level of p-S6 was significantly increased by choline. However, rapamycin significantly repressed protein level of p-mTOR and p-S6 without affecting expression of p-Akt. (A) Representative western blot bands of p-Akt and t-Akt. (B) Statistical results of $\mathrm{p}-\mathrm{Akt} / \mathrm{t}-\mathrm{Akt},{ }^{*} \mathrm{P}<0.05$ vs. sham, $\# \mathrm{P}<0.05$ vs. IR, $n=5$ each group. (C) Representative western blot bands of $p-m$ TOR and mTOR (D) Statistical results of p-mTOR/mTOR, ${ }^{*} \mathrm{P}<0.05$ vs. sham, \#P<0.05 vs. IR, $\& \mathrm{P}<0.05$ vs. Cho, $\mathrm{n}=5$ each group. (E) Representative western blot bands of p-S6. (F) Statistical results of p-S6/ $\beta$-tubulin, ${ }^{*} \mathrm{P}<0.05$ vs. IR, $\# \mathrm{P}<0.05$ vs. Cho, $\mathrm{n}=5$ each group.

mTOR expression was observed among these four groups. Furthermore, upstream signaling factor of mTOR, Akt was detected. It was indicated that protein expression of p-Akt was markedly decreased in myocardial IR, which was elevated by choline. In addition, we found that p-Akt expression in rapamycin-treated group was unchanged compared to choline 


\section{Cellular Physiology Cell Physiol Biochem 2018;45:2136-2144

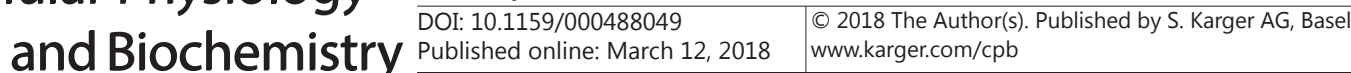 \\ Hang et al.: Choline Mitigates IR-Induced Autophagy}

treatment group (Fig. 4A, B). Moreover, protein expression of phospho-S6 (p-S6, target of mTORC1) was examined. It was found that protein level of p-S6 was stimulated in IR and further increased by choline, whereas inhibited by rapamycin (Fig. 4E, F). Collectively, these findings supported that choline attenuated IR injury by activating Akt/mTORC1.

\section{Discussion}

Autophagy is an intracellular degradation system for damaged proteins and organelles, which plays an essential role in the biological activity and governs the death and survival of cells [25]. It has been widely accepted that autophagy is a double-edged sword, which may exert both protective and harmful effects. On one hand, autophagy could promote cell survival by removing damaged proteins during nutrient deprivation and ischemia/hypoxia. On the other hand, autophagy could also trigger cell death by excessive degradation of essential cellular components or interacting with apoptotic signals. Previous studies have suggested that the role of autophagy in reperfusion is closely related to the extent of ischemia [26]. In detail, they found that in neonatal rat cardiomyocytes (NRCs) and murine hearts, autophagy was increased in response to hypoxia/reoxygenation or ischemia/reperfusion in a time-dependent manner. And the roles of rapamycin were associated with the extent of injury. In addition, beclin-1 and LC3-II are two important markers of autophagosomes, which are upregulated during the reperfusion period and signify ongoing autophagy and cellular damage. It has been uncovered that autophagy resulting from myocardial ischemia/ hypoxia is mediated by AMPK pathway. Nevertheless, myocardial IR stimulates autophagy through a beclin-1-dependent but AMPK-independent mechanism [10, 27]. Matsui et al. found that a moderate autophagy during ischemia inhibited apoptosis and promoted cell survival by activating AMPK pathway, but overactivation of autophagy during the reperfusion period aggravated myocardial injury by upregulating beclin-1 signaling [27]. Similarly, autophagy activator rapamycin also displayed discrepant effects in myocardial IR. Several studies showed the cardioprotective effect of rapamycin against IR injury [2831]. For example, it was reported that rapamycin protected against IR injury by restoring phosphorylation of STAT3 and enhancing Akt phosphorylation [30]. However, another study reported that rapamycin abolished the protective effect of hydrogen sulfide in myocardial IR by inhibiting mTOR signals [15]. In our study, rapamycin abated the beneficial effect of choline in IR heart. Based on these findings, we find that these different performances of rapamycin may result from its diverse mechanisms by regulating distinct signaling pathways as a two-edged sword. In addition, just as described in previous study [26], it was speculated that the pharmacological effect of rapamycin is closely related to autophagic activity in the heart. When the autophagy is protective, rapamycin was positive to the cardiomyocyte as the inducer of autophagy. On the contrary, if the autophagy is deterimental, rapamycin was negative to the cardiomyocyte by activating autophagy as the inhibitor of mTOR. Therefore, therapies involving the modification of autophagy should be determined according to the duration of ischaemia/reperfusion.

In this study, enhanced LC3 expression as well as accumulated autophagosomes was detected in IR heart. However, activation of LC3 at a certain time point does not indicate autophagic flux, which may be interpreted as either increased autophagosomes by activated autophagy or accumulated autophagosomes by autophagy dysfunction. So, we further detected the level of p62/SQSTM1, a protein known to be degraded by autophagy. It was found that the expression of p62 was increased in IR heart, indicating that the increased LC3 amount might be attributed to failure of autolysosome degradation. Moreover, we found that choline treatment inhibited p62 expression, which indicated that the decrease of LC3 level by choline might be attributed to promotion of autolysosome degradation. Similar results could be found in previous studies [32-35].

The dosage of rapamycin (5 mg/kg, ip) in this study was referred to previous studies $[36,37]$. And it was noted that the dosage of rapamycin range from $0.25 \mathrm{mg} / \mathrm{kg}$ to $5 \mathrm{mg} /$ $\mathrm{kg}$ in previous studies. So, in our present study, protein expression of phospho-S6 (p-S6, 
target of mTORC1) and Akt (target of mTORC2) were examined. We found that rapamycin significantly inhibited protein level of p-S6 without interfering with Akt phosphorylation. Therefore, these finding demonstrated that rapamycin selectively inhibited mTORC1.

Several previous studies have suggested that choline exerts a cardioprotective role against myocardial infarction, pathologic cardiac hypertrophy and ischemic arrhythmias. And the uncovered mechanisms include inhibition of cardiomyocyte apoptosis, decrease of calcium overload, regulation of a number of signaling pathways (eg. p38 and ERK pathways) and microRNAs (eg. miR-1, -376b-5p, -133) [38]. However, the relationship between choline and cardiomyocyte autophagy remain unclear. Therefore, here we demonstrated for the first time that choline attenuated cardiac IR-induced autophagic activity by activating Akt/ mTOR signaling pathway. Interestingly, previous study by Zhao and colleagues found that acetylcholine activated autophagy in H9c2 cells suffer from hypoxia/reoxygenation injury by AMPK pathways. In this study, they reported that acetylcholine-mediated autophagy was protective, which was abolished by autophagy inhibitor chloroqurine or Atg7 siRNA. It was displayed that the number of autophagosome and ratio of LC3-II/LC3-I were decreased in hypoxia/reoxygenation $\mathrm{H} 9 \mathrm{c} 2$ cells [39]. These data indicated that autophagic activity was distinct after different treatments although the pharmacological effect of acetylcholine was similar to choline. Besides, another explanation for this discrepancy may be the modeling difference between in vitro and in vivo studies. Furthermore, it should be emphasized that several muscarinic receptors such as $\mathrm{M}_{2}$ and $\mathrm{M}_{3}$ receptors could be activated by selective muscarinic agonist choline. Thus, whether $\mathrm{M}_{2}$ or $\mathrm{M}_{3}$ receptor was responsible for the effect of choline-mediated autophagy in myocardial IR is interesting to study in future. Studies also found that long-term rapamycin treatment may even inhibit autophagy [40]. So, it should be noted that acute effects of choline and/or rapamycin investigated in the present study may not be extrapolated to their long-term performance.

It is well known that mTOR have two isoforms: mTORC1 and mTORC2. mTORC1 activates p70S6 kinase followed by phosphorylation of ribosomal protein S6 whereas mTORC2 activates Akt by phosphorylation at Ser473. Rapamycin, as a commonly used inhibitor of mTOR, was found preferentially inhibits mTORC1 [41], although recent studies indicate that long-term treatment of rapamycin also inhibits mTORC2 [42]. Previous studies suggested that mTORC1 is the dominant complex stimulated by IR injury [43]. Consistently, in the present study, we found that rapamycin significantly inhibited p-mTOR without affecting phosphorylation of Akt expression at Ser473, suggesting that it specifically inhibited mTORC1 activity in IR hearts.

In conclusion, our study suggested that choline inhibited cardiac autophagy in IR rat heart by activating Akt/mTOR pathway, which added the new mechanistic information for the cardioprotective effect of choline and provides novel potential therapeutic targets for cardiac IR injury.

\section{Acknowledgements}

This work was supported by National Natural Science Foundation of China (81300080 to P. Hang and 81673424 to Z. Du).

\section{Disclosure Statement}

The authors declare no conflicts of interests.

\section{References}

1 Eltzschig HK, Eckle T: Ischemia and reperfusion--from mechanism to translation. Nat Med 2011;17:13911401.

2 Altamirano F, Wang ZV, Hill JA: Cardioprotection in ischaemia-reperfusion injury: novel mechanisms and clinical translation. J Physiol 2015;593:3773-3788.

\section{KARGER}




\section{Cellular Physiology Cell Physiol Biochem 2018;45:2136-2144 \begin{tabular}{ll|l} 
and Biochemistry Published online: March 12, 2018 & $\begin{array}{l}\text { (c) } 2018 \text { The Author(s). Published by S. Karger AG, Basel } \\
\text { www.karger.com/cpb }\end{array}$
\end{tabular}}

Hang et al.: Choline Mitigates IR-Induced Autophagy

3 Oerlemans MI, Koudstaal S, Chamuleau SA, de Kleijn DP, Doevendans PA, Sluijter JP: Targeting cell death in the reperfused heart: pharmacological approaches for cardioprotection. Int J Cardiol 2013;165:410-422.

4 Chen-Scarabelli C, Agrawal PR, Saravolatz L, Abuniat C, Scarabelli G, Stephanou A, Loomba L, Narula J, Scarabelli TM, Knight R: The role and modulation of autophagy in experimental models of myocardial ischemia-reperfusion injury. J Geriatr Cardiol 2014;11:338-348.

5 Ma S, Wang Y, Chen Y, Cao F: The role of the autophagy in myocardial ischemia/reperfusion injury. Biochim Biophys Acta 2015;1852:271-276.

6 Mizushima N, Levine B, Cuervo AM, Klionsky DJ: Autophagy fights disease through cellular self-digestion. Nature 2008;451:1069-1075.

-7 Li Q Dong QT, Yang YJ, Tian XQ Jin C, Huang PS, Jiang LP, Chen GH: AMPK-mediated cardioprotection of atorvastatin relates to the reduction of apoptosis and activation of autophagy in infarcted rat hearts. Am J Transl Res 2016;8:4160-4171.

-8 Zheng Y, Gu S, Li X, Tan J, Liu S, Jiang Y, Zhang C, Gao L, Yang HT: Berbamine postconditioning protects the heart from ischemia/reperfusion injury through modulation of autophagy. Cell Death Dis 2017;8:e2577.

-9 Xue R, Zeng J, Chen Y, Chen C, Tan W, Zhao J, Dong B, Sun Y, Dong Y, Liu C: Sestrin 1 ameliorates cardiac hypertrophy via autophagy activation. J Cell Mol Med 2017;21:1193-1205.

10 Ma X, Liu H, Foyil SR, Godar RJ, Weinheimer CJ, Hill JA, Diwan A: Impaired autophagosome clearance contributes to cardiomyocyte death in ischemia/reperfusion injury. Circulation 2012;125:3170-3181.

11 Ma X, Liu H, Foyil SR, Godar RJ, Weinheimer CJ, Diwan A: Autophagy is impaired in cardiac ischemiareperfusion injury. Autophagy 2012;8:1394-1396.

12 Dhesi P, Tehrani F, Fuess J, Schwarz ER: How does the heart (not) die? The role of autophagy in cardiomyocyte homeostasis and cell death. Heart Fail Rev 2010;15:15-21.

13 Gao L, Guo Y, Liu X, Shang D, Du Y: KLF15 protects against isoproterenol-induced cardiac hypertrophy via regulation of cell death and inhibition of Akt/mTOR signaling. Biochem Biophys Res Commun 2017;487:22-27.

14 Chen Y, Zhou X, Qiao J, Bao A: MiR-142-3p overexpression increases chemo-sensitivity of NSCLC by inhibiting HMGB1-mediated autophagy. Cell Physiol Biochem 2017;41:1370-1382.

-15 Xiao J, Zhu X, Kang B, Xu J, Wu L, Hong J, Zhang Y, Ni X, Wang Z: Hydrogen sulfide attenuates myocardial hypoxia-reoxygenation injury by inhibiting autophagy via mTOR activation. Cell Physiol Biochem 2015;37:2444-2453.

16 Wang ZG, Li H, Huang Y, Li R, Wang XF, Yu LX, Guang XQ, Li L, Zhang HY, Zhao YZ, Zhang C, Li XK, Wu RZ, Chu MP, Xiao J: Nerve growth factor-induced Akt/mTOR activation protects the ischemic heart via restoring autophagic flux and attenuating ubiquitinated protein accumulation. Oncotarget 2017;8:5400-5413.

17 Wang ZG, Wang Y, Huang Y, Lu Q Zheng L, Hu D, Feng WK, Liu YL, Ji KT, Zhang HY, Fu XB, Li XK, Chu MP, Xiao J: bFGF regulates autophagy and ubiquitinated protein accumulation induced by myocardial ischemia/ reperfusion via the activation of the PI3K/Akt/mTOR pathway. Sci Rep 2015;5:9287.

-18 Yang B, Lin H, Xu C, Liu Y, Wang H, Han H, Wang Z: Choline produces cytoprotective effects against ischemic myocardial injuries: evidence for the role of cardiac $\mathrm{m} 3$ subtype muscarinic acetylcholine receptors. Cell Physiol Biochem 2005;16:163-174.

-19 Wang S, Han HM, Jiang YN, Wang C, Song HX, Pan ZY, Fan K, Du J, Fan YH, Du ZM, Liu Y: Activation of cardiac M3 muscarinic acetylcholine receptors has cardioprotective effects against ischaemia-induced arrhythmias. Clin Exp Pharmacol Physiol 2012;39:343-349.

20 Zhao J, Su Y, Zhang Y, Pan Z, Yang L, Chen X, Liu Y, Lu Y, Du Z, Yang B: Activation of cardiac muscarinic M3 receptors induces delayed cardioprotection by preserving phosphorylated connexin43 and up-regulating cyclooxygenase-2 expression. Br J Pharmacol 2010;159:1217-1225.

21 Wang S, Han HM, Pan ZW, Hang PZ, Sun LH, Jiang YN, Song HX, Du ZM, Liu Y: Choline inhibits angiotensin II-induced cardiac hypertrophy by intracellular calcium signal and p38 MAPK pathway. Naunyn Schmiedebergs Arch Pharmacol 2012;385:823-831.

-22 Hang P, Zhao J, Sun L, Li M, Han Y, Du Z, Li Y: Brain-derived neurotrophic factor attenuates doxorubicininduced cardiac dysfunction through activating Akt signalling in rats. J Cell Mol Med 2017;21:685-696.

23 Huang W, Tian SS, Hang PZ, Sun C, Guo J, Du ZM: Combination of microRNA-21 and microRNA-146a attenuates cardiac dysfunction and apoptosis during acute myocardial infarction in mice. Mol Ther Nucleic Acids 2016;5:e296. 


\section{Cellular Physiology Cell Physiol Biochem 2018;45:2136-2144 \begin{tabular}{ll|l} 
and Biochemistry Published online: March 12, 2018 & $\begin{array}{l}\text { (C) } 2018 \text { The Author(s). Published by S. Karger AG, Basel } \\
\text { www.karger.com/cpb }\end{array}$
\end{tabular}}

Hang et al.: Choline Mitigates IR-Induced Autophagy

24 Hang P, Zhao J, Cai B, Tian S, Huang W, Guo J, Sun C, Li Y, Du Z: Brain-derived neurotrophic factor regulates TRPC3/6 channels and protects against myocardial infarction in rodents. Int J Biol Sci 2015;11:536-545.

25 Levine B, Klionsky DJ: Development by self-digestion: molecular mechanisms and biological functions of autophagy. Dev Cell 2004;6:463-477.

-26 Xu Q, Li X, Lu Y, Shen L, Zhang J, Cao S, Huang X, Bin J, Liao Y: Pharmacological modulation of autophagy to protect cardiomyocytes according to the time windows of ischaemia/reperfusion. Br J Pharmacol. 2015;172:3072-3085.

-27 Matsui Y, Takagi H, Qu X, Abdellatif M, Sakoda H, Asano T, Levine B, Sadoshima J: Distinct roles of autophagy in the heart during ischemia and reperfusion: roles of AMP-activated protein kinase and Beclin 1 in mediating autophagy. Circ Res 2007;100:914-922.

-28 Filippone SM, Samidurai A, Roh SK, Cain CK, He J, Salloum FN, Kukreja RC, Das A: Reperfusion therapy with rapamycin attenuates myocardial infarction through activation of AKT and ERK. Oxid Med Cell Longev 2017;2017:4619720

29 Das A, Salloum FN, Filippone SM, Durrant DE, Rokosh G, Bolli R, Kukreja RC: Inhibition of mammalian target of rapamycin protects against reperfusion injury in diabetic heart through STAT3 signaling. Basic Res Cardiol 2015;110:31.

-30 Das A, Salloum FN, Durrant D, Ockaili R, Kukreja RC: Rapamycin protects against myocardial ischemiareperfusion injury through JAK2-STAT3 signaling pathway. J Mol Cell Cardiol 2012;53:858-869.

-31 Khan S, Salloum F, Das A, Xi L, Vetrovec GW, Kukreja RC: Rapamycin confers preconditioning-like protection against ischemia-reperfusion injury in isolated mouse heart and cardiomyocytes. J Mol Cell Cardiol 2006;41:256-264.

32 Song H, Yan C, Tian X, Zhu N, Li Y, Liu D, Liu Y, Liu M, Peng C, Zhang Q Gao E, Han Y: CREG protects from myocardial ischemia/reperfusion injury by regulating myocardial autophagy and apoptosis. Biochim Biophys Acta 2017;1863:1893-1903.

33 Yao T, Ying X, Zhao Y, Yuan A, He Q, Tong H, Ding S, Liu J, Peng X, Gao E, Pu J, He B: Vitamin D receptor activation protects against myocardial reperfusion injury through inhibition of apoptosis and modulation of autophagy. Antioxid Redox Signal 2015;22:633-650.

-34 Xie H, Xu Q, Jia J, Ao G, Sun Y, Hu L, Alkayed NJ, Wang C, Cheng J: Hydrogen sulfide protects against myocardial ischemia and reperfusion injury by activating AMP-activated protein kinase to restore autophagic flux. Biochem Biophys Res Commun 2015;458:632-638.

-35 Fan G, Yu J, Asare PF, Wang L, Zhang H, Zhang B, Zhu Y, Gao X: Danshensu alleviates cardiac ischaemia/ reperfusion injury by inhibiting autophagy and apoptosis via activation of mTOR signalling. J Cell Mol Med 2016;20:1908-1919.

-36 Zhou Y, Fang H, Lin S, Shen S, Tao L, Xiao J, Li X: Qiliqiangxin protects against cardiac ischemia-reperfusion injury via activation of the mTOR pathway. Cell Physiol Biochem 2015;37(2):454-464.

37 Yang SS, Liu YB, Yu JB, Fan Y, Tang SY, Duan WT, Wang Z, Gan RT, Yu B: Rapamycin protects heart from ischemia/reperfusion injury independent of autophagy by activating PI3 kinase-Akt pathway and mitochondria K(ATP) channel. Pharmazie 2010;65:760-765.

38 Hang P, Zhao J, Qi J, Wang Y, Wu J, Du Z: Novel insights into the pervasive role of M3 muscarinic receptor in cardiac diseases. Curr Drug Targets 2013;14:372-377.

39 Miao Y, Zhou J, Zhao M, Liu J, Sun L, Yu X, He X, Pan X, Zang W: Acetylcholine attenuates hypoxia/ reoxygenation-induced mitochondrial and cytosolic ROS formation in H9c2 cells via M2 acetylcholine receptor. Cell Physiol Biochem 2013;31:189-198.

40 Kurdi A, De Doncker M, Leloup A, Neels H, Timmermans JP, Lemmens K, Apers S, De Meyer GRY, Martinet W: Continuous administration of the mTORC1 inhibitor everolimus induces tolerance and decreases autophagy in mice. Br J Pharmacol 2016;173(23):3359-3371.

41 Hennig M, Fiedler S, Jux C, Thierfelder L, Drenckhahn JD: Prenatal mechanistic target of rapamycin complex 1 (m TORC1) inhibition by rapamycin treatment of pregnant mice causes intrauterine growth restriction and alters postnatal cardiac growth, morphology, and function. J Am Heart Assoc 2017;6(8).

42 Sarbassov DD, Ali SM, Sengupta S, Sheen JH, Hsu PP, Bagley AF, Markhard AL, Sabatini DM: Prolonged rapamycin treatment inhibits mTORC2 assembly and Akt/PKB. Mol Cell 2006;22:159-168.

-43 Aoyagi T, Kusakari Y, Xiao CY, Inouye BT, Takahashi M, Scherrer-Crosbie M, Rosenzweig A, Hara K, Matsui T: Cardiac mTOR protects the heart against ischemia-reperfusion injury. Am J Physiol Heart Circ Physiol 2012;303:H75-H85. 